\author{
Was ist neu in der medikamentösen Therapie? Wir halten Sie auf dem Laufenden \\ mit Berichten von Kongressen und Symposien der pharmazeutischen Industrie.
}

\title{
Schilddrüsenerkrankungen
}

\section{Bei Struma primär medikamentös behandeln}

_ Eine Struma ist auch heutzutage noch eine Volkskrankheit, sagte PD Dr. Dr. Natascha Döbert, Praxis für Nuklearmedizin, Darmstadt. Laut der Papillon-Studie haben über 30\% der Bundesbürger morphologische Auffälligkeiten der Schilddrüse, erläuterte die Fachärztin für Nuklearmedizin.

Welche Art der Strumatherapie zum Einsatz kommen soll, hängt nach Döbert im Wesentlichen von folgenden Kriterien ab:

- Ausmaß der Schilddrüse, anatomische Umgebung,

- Knotendignität/-funktionalität (Malignomverdacht, Autonomie)

- Symptomatik (lokal, symptomatisch)

- Hormonlage,

- Antikörperprofil und

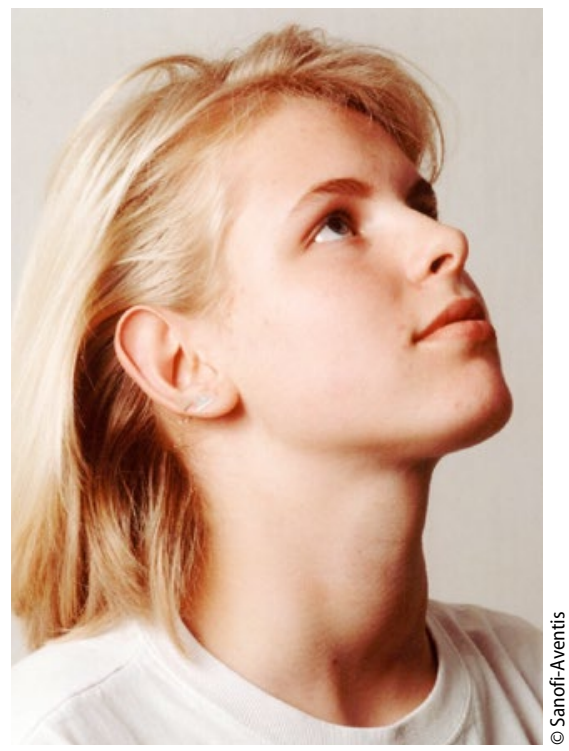

Eine Struma kann auch bei jungen Menschen auftreten.
- Patientenalter (kardiovaskuläre Risikofaktoren).

Primäres Ziel sei die medikamentöse Behandlung. Hier gehe es darum, weitere Knoten zu verhindern und das Volumen bestehender Knoten zu reduzieren. Dies könne dann auch zu einer Reduktion klinischer Symptome (etwa Globusgefühl) führen, erläuterte Döbert.

Bei einer Rezidivstruma oder begleitender Autonomie ist die Radiojodtherapie zu erwägen. Eine Operation sollte vor allem ausgeprägteren Strumen vorbehalten bleiben. Auch bei lokalen Kompressionen oder Malignomverdacht bleibe nur der operative Eingriff, so die Nuklearmedizinerin. Bei benignen großen Strumen, symptomatischen Knoten und Rezidivstruma sind neuere thermoablative Verfahren zu erwägen (Mikrowellentherapie, Radiofrequenzablation, hochfokussierter Ultraschall).

Die heutige medikamentöse Therapieempfehlung basiert auf der randomisierten, placebokontrollierten LISA-Studie [J Clin Endocrinol Metab. 2011;96: 2786-95], in der sich gezeigt hat, dass die Kombination aus LT4 (L-Thyroxin) und Jod die Therapie der Wahl ist. Unter LT-4 bildet sich die Hypertrophie der Follikelzellen zurück, Jod führt zu einer Hemmung der Zellproliferation und damit zu einem Rückgang der Hyperplasie.

Vor der Kombinationsbehandlung sollte auf jeden Fall eine Autonomie und Schilddrüsenantikörper ausgeschlossen werden, erinnerte Döbert. Bei einer nichtstoffwechselrelevanten Autonomie und/ oder bei Schilddrüsenantikörpern ist eine jodfreie L-Thyroxin-Medikation angezeigt. Regelmäßige Therapiekontrollen sind erforderlich.
Bei Menschen über 70 sollten höhere TSH-Werte (Zielwert ca. 2-6 mU/l) angestrebt und auch langsamer aufdosiert werden. Hier könne auch eine halbierte 25er-L-Thyroxin-Tablette genommen und anschließend aufdosiert werden, so Döbert. Wichtig ist, dass der Patient die Behandlung gut verträgt und dass keine kardiovaskulären Risikofaktoren vorhanden sind. Die Aufdosierung ist zudem abhängig vom Schweregrad der Struma und vom Gesamtzustand des $\mathrm{Pa}$ tienten.

\section{Christina Ott}

- Schilddrüsen-Update 2016; Darmstadt, April 2016 (Veranstalter Sanofi/Henning)

\section{Kurz notiert}

Mit Opioiden in den Urlaub $\rightarrow$ Für Opioidpatienten ist eine kontinuierliche Therapie auch im Urlaub wichtig. Eine frühzeitige Beratung durch Arzt oder Apotheker hilft Patienten, ihre Reise entspannt anzutreten. Als meistverordneter Anbieter von Fentanylpflastern hat sich $1 \mathrm{~A}$ Pharma dieses Themas angenommen und das neue „Einfach-Hilfreich"-Paket "Reisen mit Opioiden" geschnürt. Enthalten sind praktische Tipps für die Urlaubsvorbereitung, ein mehrsprachiger Opioidausweis, zwei Bescheinigungen für das Mitführen von Beträubungsmitteln innerhalb bzw. außerhalb des Schengenraums sowie ein Umschlag, in dem die Patienten ihre medizinischen Reiseunterlagen mit sich führen können. In einem „Einfach-Hilfreich“-Paket sind die Materialien in fünffacher Ausfertigung enthalten. Ärzte und Apotheker können das Paket kostenlos per Fax (08006131111) und unter www.1apharma.de/service bestellen.

Red.

- Nach Informationen von 1 A Pharma 